\title{
Mechanisms of Chikungunya virus disease informed by Ross River virus research
}

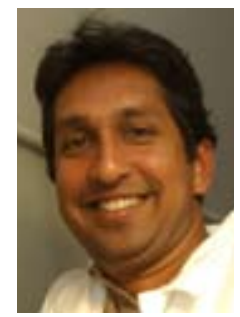

"The immunopathological mechanisms responsible for CHIKV disease are poorly understood ... much can be learnt about alphaviral disease mechanisms by drawing on current RRV studies and disease models."

Umang Srivastava*, Michelle Nelson*, Yung-Chang Su \& Suresh Mahalingam ${ }^{\dagger}$

†Author for correspondence $=$ Faculty of Applied Science, University of Canberra, Canberra, ACT 2601, Australia m Tel. : +61 262012368 max: +61 262015727 msuresh.mahalingam@canberra.edu.au *Both authors contributed equally

Chikungunya virus (CHIKV) was first isolated in Tanzania in 1953 [1]. Since then, CHIKV outbreaks have occurred sporadically in SubSaharan Africa [2], southeast Asia [3] and India [4]. Most recently, in 2005/2006, a CHIKV outbreak was reported in a number of islands in the western Indian Ocean, which has since spread into India, giving rise to the largest CHIKV epidemic on record [5]. Reports of CHIKV cases in Italy, a more temperate environment, have suggested the potential for this virus to spread worldwide [6]. CHIKV, an Alphavirus belonging to the Togaviridae family, is maintained by transmission cycles between mosquito vectors and vertebrate hosts. Whilst CHIKV disease is generally not fatal, it can cause debilitating symptoms including fever, rash, headache, myalgia and arthralgia. Some more severe cases have been described, for example, in neonates where infection has been found to involve the CNS [7]. As yet, there are no specific treatments available for CHIKV disease, although analgesics and nonsteroidal anti-inflammatory drugs can be given for symptomatic relief.

\footnotetext{
"Reports of CHIKV cases in Italy, a more temperate environment, have suggested the potential for this virus to spread worldwide."
}

CHIKV is closely related to Ross River virus (RRV), an Australasian Alphavirus responsible for outbreaks of infectious polyarthritis in Australia and the western Pacific Islands. Both CHIKV and RRV belong to the Semliki Forest virus group, a phylogenetically related group of alphaviruses [8]. RRV disease exhibits symptoms similar to that seen for CHIKV disease, including arthralgia, rash, lethargy and myalgia [8]. Both diseases also have a similar incubation period, duration and can, on rare occasions, cause complications involving the CNS, such as encephalitis [9,10]. RRV, like CHIKV, primarily targets skeletal muscles and joints [11,12], causing a range of symptoms from tenderness and minor movement restriction to severe swelling, effusion and redness.

\section{Proposed mechanism of disease}

The immunopathological mechanisms responsible for CHIKV disease are poorly understood, primarily owing to the lack of suitable animal models of disease. However, much can be learnt about alphaviral disease mechanisms by drawing on current RRV studies and disease models. Not only could these studies provide an insight into the mechanisms of CHIKV disease, they may also provide essential clues for the development of a much needed mouse model of CHIKV disease that mimics the adult human arthritic disease. A recent study has established a mouse model for CHIKV disease using neonatal and type-1 interferon (IFN)-deficient mice [12]. This model mimics the more severe cases of CHIKV, resulting in flaccid paralysis, lethargy, viral dissemination to the CNS and sometimes mortality. Mice with partial deficiency in type-1 IFN provided a model for benign CHIKV infection, where no lethality or mortality was observed but infectious virus was obtained from various tissues. Wild-type mice, however, failed to exhibit any signs of infection. Unfortunately, these mouse models do not mimic many aspects of the human disease as the models do not show prominent limb weakness and inflammatory response in bone and joint tissues. Clearly, more research is needed to develop an adult mouse model appropriate for this form of the disease.
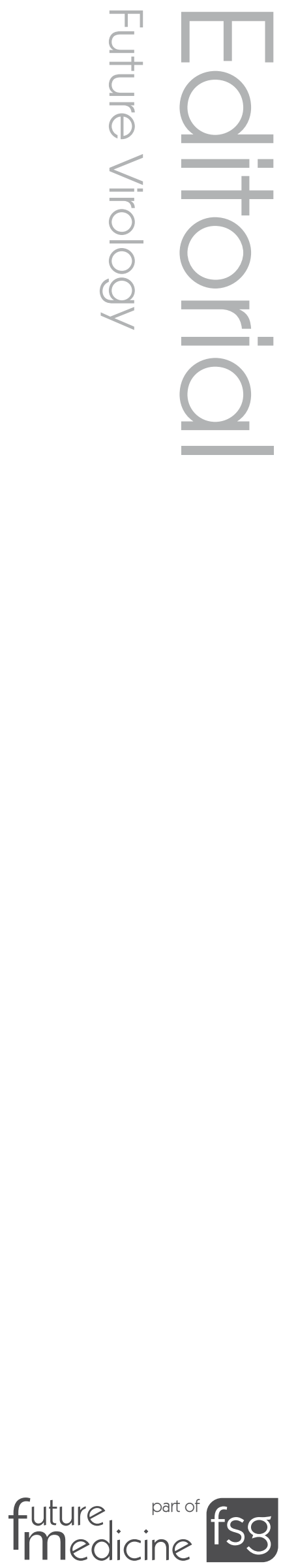
The initial studies involving a mouse animal model for CHIKV infection have similarities to early RRV studies. For example, in 1973, studies into RRV infection in outbred mice showed a similar spread of virus through a wide range of tissues, including the CNS, although no evidence of RRV replication or RRV-mediated pathology was found in the joint tissues [13,14]. The targeting of connective tissue of joints and skeletal muscles have been identified as sites of viral replication for both CHIKV [12] and RRV within the respective mouse models. Similar to what was seen for CHIKV in these and previous studies [15], we have found that RRV is unable to replicate in mouse blood leukocytes and primary human monocytes, except when infection is performed in the presence of subneutralizing concentrations of anti-RRV antibody through a process known as antibody-dependent enhancement of infection [Rulli N, Musso C, Mahalingam S, Unpublished data].

The early RRV mouse models were originally thought to be unsuitable models for human RRV-induced arthritic disease due to the lack of RRV-mediated pathology in the joint tissues. After further development, however, a RRV disease model in outbred mice was established, with characteristics that clearly mimicked that of the human disease [16]. Mice developed arthritic symptoms in the hind limbs at 7-9 days postinfection, with a dramatic infiltration of monocytes and macrophages into the hind-limb muscle, causing associated disruption to the muscle structure. Full recovery of mice was seen after approximately 25 days postinfection. This model was successfully reproduced using inbred mice, resulting in similar clinical features and pathology [11]. Since the newly developed CHIKV disease mouse models also do not mimic the less severe form of CHIKV disease, showing either severe symptoms or not producing detectable arthritic symptoms, the lessons learnt from the establishment of an arthritic RRV mouse model could provide valuable clues for the development of a more appropriate mouse model.

The role of the macrophage in alphaviral immunopathogenesis has been identified through studies involving the mouse model of RRV disease [16]. Amelioration of RRV-induced arthritis and myositis was found following depletion of macrophages from the mouse prior to infection [16], and macrophage-derived proinflammatory mediators have been implicated in the pathogenesis of RRV disease [17]. Synovial effusions from RRV patients are also known to have high levels of activated macrophages and monocytes, confirming the physiological relevance of these results $[18,19]$. In addition, RRV can persist in macrophages for long periods [20], suggesting a role for these cells in RRV persistence in addition to pathogenesis. These observations may also be relevant to the immunopathological processes that lead to arthritic symptoms following CHIKV infection and may provide a key starting point for further investigations into the mechanisms involved in CHIKV disease.

In summary, the knowledge gained from RRV studies and animal models can be used to understand the mechanisms behind alphaviral disease in general. Specifically, a greater understanding could be gained about the pathogenesis of CHIKV disease by adapting our understanding of RRV disease.

\section{Financial \& competing interests disclosure \\ The authors have no relevant affiliations or financial involvement with any organization or entity with a financial interest in or financial conflict with the sub- ject matter or materials discussed in the manuscript. This includes employment, consultancies, honoraria, stock ownership or options, expert testimony, grants or patents received or pending, or royalties. \\ No writing assistance was utilized in the production of this manuscript.}

\section{Bibliography}

1. Ross RW: The Newala Epidemic. III. The virus: isolation, pathogenic properties and relationship to the epidemic. J. Hygiene 54(2), 177-191 (1956).

2. Pastorino B, Muyembe-Tamfum JJ, Bessaud $\mathrm{M}$ et al.: Epidemic resurgence of Chikungunya virus in democratic Republic of the Congo: identification of a new central African strain. J. Med. Virology 74(2), 277-282 (2004).
3. Lam SK, Chua KB, Hooi PS: Chikungunya infection: emerging disease in Malaysia. Southeast Asian J. Trop. Med. Public Health 32(2), 447-451 (2001).

4. Shah KV, Gibbs CJJ, Banerjee G: Virological investigation of the epidemic of haemorrhagic fever in Calcutta: isolation of three strains of Chikungunya virus. Indian J. Med. Res. 52, 676-683 (1964).

D. Enserink M: Infectious diseases. Massive outbreak draws fresh attention to little-known virus. Science 311, 1085 (2006).
6. Rezza G, Nicoletti L, Angelini R et al.: CHIKV study group. Infection with Chikungunya virus in Italy: an outbreak in a temperate region. Lancet 370, 1840-1846 (2007).

Gerardin P, Barau G, Michault A et al.: Multidisciplinary prospective study of mother-to-child Chikungunya virus infections on the Island of La Reunion. PLoS Med. 5(3), e60 (2008).

8. Johnston RE, Peters CJ: Alphaviruses. In: Fields Virology. Fields BN et al. (Eds). Lippincott-Raven Publishers, Philadelphia, PA, USA, 843-898 (1996). 
9. Bessaud M, Peyrefitte CN, Pastorino BA et al:: Chikungunya virus strains, Reunion Island outbreak [letter]. Emerging Infect. Dis. 12(10), 1604-1606 (2006).

10. Scrimgeour EM, Aaskov JG, Leonard RM: Ross River virus arthritis in Papua New Guinea. Trans. R. Soc. Trop. Med. Hyg. 81(5), 833-834 (1987).

11. Morrison TE, Whitmore AC, Shabman RS, Lidbury BA, Mahalingam S, Heise MT: Characterization of Ross River virus tropism and virus-induced inflammation in a mouse model of viral arthritis and myositis. J. Virol. 80(2), 737-749 (2006).

12. Couderc T, Chrétien F, Schilte C et al:

A mouse model for Chikungunya: young age and inefficient type-I interferon signaling are risk factors for severe disease. PLoS Pathogens 4(2), e29 (2008).

13. Mims CA, Murphy FA, Taylor WP, Marshall ID: Pathogenesis of Ross River virus infection in mice. I. Ependymal infection, cortical thinning, and hydrocephalus. J. Infect. Dis. 127(2), 121-128 (1973).

14. Murphy FA, Taylor WP, Mims CA, Marshall ID: Pathogenesis of Ross River virus infection in mice. II. Muscle, heart, and brown fat lesions. J. Infect. Dis. 127(2), 129-138 (1973).
15. Sourisseau M, Schilte C, Casartelli N et al:: Characterization of reemerging Chikungunya virus. PLoS Pathogens 3, e89 (2007).

16. Lidbury BA, Simeonovic C, Maxwell GE, Marshall ID, Hapel AJ: Macrophage induced muscle pathology results in morbidity and mortality for Ross River virus-infected mice. J. Infect. Dis. 181, 27-34 (2000).

17. Lidbury BA, Rulli NE, Suhrbier A et al.: Macrophage-derived pro-inflammatory factors contribute to the development of arthritis and myositis following infection with an arthrogenic alphavirus. J. Infect. Dis. 197, 1585-1593 (2008).

18. Fraser JR, Cunningham AL, Clarris BJ, Aaskov JG, Leach R: Cytology of synovial effusions in epidemic polyarthritis. Aust. $N$. Z. J. Med. 11, 168-173 (1981).

19. Fraser JR, Becker GJ: Mononuclear cell types in chronic synovial effusions of Ross River virus disease. Aust. N. Z. J. Med. 14, 505-506 (1984).

20. Way SJ, Lidbury BA, Banyer JL: Persistent Ross River virus infection of murine macrophages: an in vitro model for the study of viral relapse and immune modulation during long-term infection. Virology 301, 281-292 (2002).

\section{Affiliations}

- Umang Srivastava

Virus \& Inflammation Research Group, Centre for Biomolecular \& Chemical Sciences, Faculty of Applied Science, University of Canberra, Canberra, 2601, Australia

- Michelle Nelson

Virus \& Inflammation Research Group, Centre for Biomolecular \& Chemical Sciences, Faculty of Applied Science, University of Canberra,

Canberra, 2601,

Australia

- Yung-Chang Su

Virus \& Inflammation Research Group,

Centre for Biomolecular \& Chemical Sciences, Faculty of Applied Science, University of Canberra,

Canberra, 2601,

Australia

\section{- Suresh Mahalingam}

Faculty of Applied Science, University of Canberra, Canberra, ACT 2601, Australia

Tel: + +61 262012 368;

Fax: +61 262015727

suresh.mahalingam@canberra.edu.au 